\section{EXHIBITION}

\section{Cultures in the capital}

\section{Jane Rees}

Our skin senses the world and projects our persona. This month in Liverpool, UK, 17 international artists incorporate skin and tissue cultures - either metaphorical or real - into artworks that reflect on how technologies may influence our future. The exhibition is part of Liverpool's European Capital of Culture 2008 celebrations.

The show is not for the squeamish. Examples of xenotransplantation, stemcell research and genetic modification are among the exhibits at sk-interfaces at the Foundation for Art and Creative Technology (FACT). Face-transplant surgeon Peter Butler of the Royal Free Hospital in London, who opened the exhibition, suggested that using the techniques of bioscience to create works of art allows us to "dream about possibilities".

One work asks whether we can grow leather without harming animals. In Victimless Leather, artists Oron Catts and Ionat Zurr have formed three tiny jackets by growing a mouse cell-culture over a polymer mould. The polymer biodegrades over time, leaving the minute skin garments. Is such technology 'victim'free? Not necessarily - cell-culture medium that includes fetal bovine serum is required to keep the cells alive.

Other works are equally bold. Sitting gruesomely in jam jars are two artists' co-cultured epidermal cells, which have been grafted onto pig derma and then tattooed with images of endangered species. To fully appreciate this art, produced by Marion Laval-Jeantet and Benoit Mangin, a collector may choose to have it xenotransplanted onto his or her own skin.

The performance artist Orlan (famous for her 'Carnal Art' plastic surgery interventions in the 1990s) has patched together a Harlequin coat from a mixture of her own cells, animal cells and, most controversially, cells from a 12-week-old human fetus of African origin, purchased over the Internet. She asks us to contemplate our hybrid origins and even our concept of species.

The artist Stelarc has sewn an ear-shaped prosthesis onto his left forearm, constructed from silicon scaffold and stem cells; the surgery is shown on continuous video loop on a large screen. He hopes to have a microphone and Bluetooth transmitter installed so that his extra ear becomes an 'Internet organ' for the body.

Legislation affects art as much as science in Jun Takita's Light Only Light, a model of the artist's brain covered with 'transgenic' bioluminescent moss - alas, with no detectable

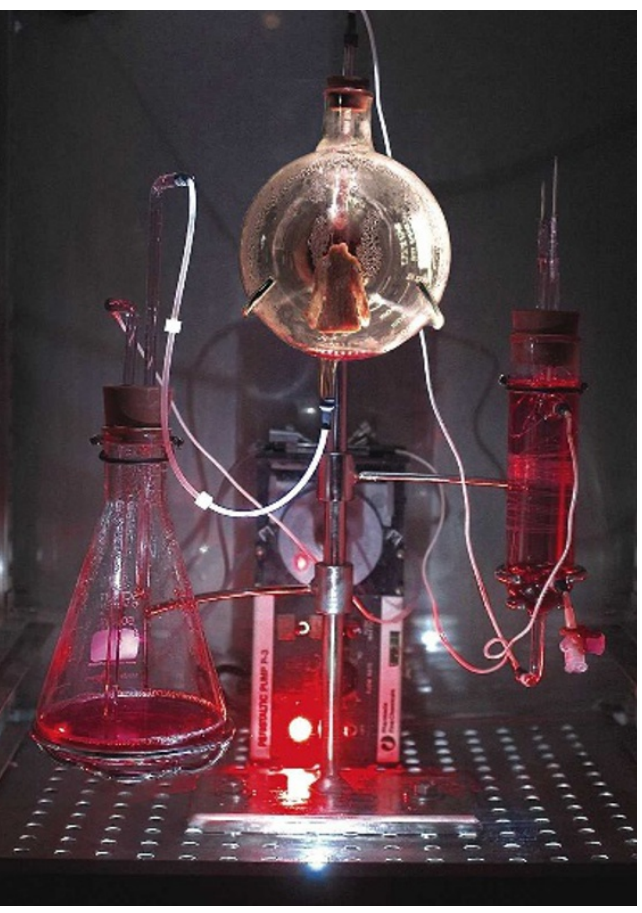

A tiny leather jacket grown in the lab from mouse cells.

glow. A notice states: "Due to uncertain legal status of displaying genetically modified plants, non-transgenic moss is shown here."

A brave exhibition, sk-interfaces should provoke reaction, thought and discussion. Conceding that some will find it "distasteful", north-west regional director of public health John Ashton, says: "It begins to raise the question about whether challenging art of this type has any limits." However, John Hunt, a clinical engineer from the University of Liverpool who helped to produce Victimless Leather, hopes the exhibition will "encourage people to think about the concepts and boundaries" of clinical techniques so they can personally decide whether there are ethical issues.

Sk-interfaces is the first of three exhibitions to be held at FACT as part of its Human Futures programme, celebrating Liverpool's stint as European Capital of Culture. Science events elsewhere in the city this year include the opening of the Victoria Gallery and Museum housing artefacts such as a prototype Geiger counter owned by James Chadwick, and clay sculptor Rod Harris's residency at the University of Liverpool for Exploring Creativity in Engineering and Science with Sculpture.

Jane Rees is research communication adviser at the University of Liverpool, Liverpool L69 3GL, UK.

\footnotetext{
Sk-interfaces runs at the Foundation for Art and Creative Technology in Liverpool until 30 March. See www.liverpool08.com for other European Capital of Culture events.
}

\title{
Text book for space shakers
}

\author{
Gravitational Waves: Volume 1: Theory \\ and Experiments \\ by Michele Maggiore \\ Oxford University Press: 2007. 572 pp. \$90
}

\section{Neil Turok}

Gravitational waves are ripples in space-time that travel through space at the speed of light. They are emitted by astrophysical sources such as binary stars or colliding black holes, or by violent phenomena such as cosmic strings or inflation in the very early Universe. Because they affect matter only weakly, gravitational waves are very hard to detect. To do so we need large instruments - interferometers several kilometres on a side - with extremely sensitive quantum-limited detectors, only recently available. The very fact that gravity couples so weakly to matter means that gravitational waves travel almost unimpeded through any material between us and the source of the gravitational waves. So they can provide a unique, direct view of some of the most dramatic cosmic events, including black-hole collisions or even the Big Bang itself.

At least that's the theory. Gravitational waves are confidently predicted on the basis of general relativity, but we've not been able to directly measure them so far. Their detection will be a scientific triumph, opening a brand new window onto the Universe. Over the past three decades, the technology of gravitationalwave detection has been steadily advancing, and sophisticated instruments are now operating in Italy, Japan, Germany and the United States. Versions coming online within a few years should reach the sensitivities required to see the gravitational waves expected from distant collisions involving black holes or neutron stars. Far more ambitious space-based experiments such as the Laser Interferometric Space Antenna (LISA) - a mission jointly led by the European Space Agency and NASA - are also planned for launch within the next decade. A growing community of experimentalists and theorists is forming, focused on the huge challenges of instrumental design and data analysis.

Students and experienced researchers will welcome Michele Maggiore's timely and authoritative new text book of classic results, detailed applications to specific gravitational wave sources, advanced mathematical methods and experimental issues. The first of two planned volumes, Gravitational Waves provides a thorough grounding in the theory of gravitational wave emission and a description of the practical techniques involved. It gathers many scattered results from a wide literature into a detailed, coherent, pedagogical review. The second volume, still in preparation, will cover 